\title{
Small bowel carcinomas in celiac or Crohn's disease: distinctive histophenotypic, molecular and histogenetic patterns
}

\author{
Alessandro Vanoli ${ }^{1,2,3,17}$, Antonio Di Sabatino ${ }^{4,17}$, Michele Martino ${ }^{4}$, Catherine Klersy ${ }^{5}$, \\ Federica Grillo ${ }^{6}$, Claudia Mescoli ${ }^{7}$, Gabriella Nesi $^{8}$, Umberto Volta ${ }^{9}$, Daniele Fornino ${ }^{1,2}$, \\ Ombretta Luinetti ${ }^{1,2}$, Paolo Fociani ${ }^{10}$, Vincenzo Villanacci ${ }^{11}$, Francesco P D’Armiento ${ }^{12}$, \\ Renato Cannizzaro $^{13}$, Giovanni Latella ${ }^{14}$, Carolina Ciacci ${ }^{15}$, Livia Biancone ${ }^{16}$, \\ Marco Paulli ${ }^{1,2}$, Fausto Sessa ${ }^{3}$, Massimo Rugge ${ }^{7}$, Roberto Fiocca ${ }^{6}$, Gino R Corazza ${ }^{4}$ and \\ Enrico Solcia ${ }^{1,2}$
}

${ }^{1}$ Department of Molecular Medicine, University of Pavia, Pavia, Italy; ${ }^{2}$ Pathology Unit, IRCCS San Matteo Hospital, Pavia, Italy; ${ }^{3}$ Department of Surgical and Morphological Sciences, University of Insubria, Varese, Italy; ${ }^{4}$ Department of Internal Medicine, IRCCS San Matteo Hospital, University of Pavia, Pavia, Italy; ${ }^{5}$ Biometry and Statistics Service, IRCCS San Matteo Hospital, Pavia, Italy; ${ }^{6}$ Pathology Unit, Department of Surgical and Diagnostic Sciences, San Martino/IST University Hospital, Genova, Italy; ${ }^{7}$ Pathology Unit, Department of Medicine, University of Padua, Padua, Italy; ${ }^{8}$ Division of Pathological Anatomy, Department of Surgery and Translational Medicine, University of Florence, Florence, Italy; ${ }^{9}$ Division of Gastroenterology, Sant'Orsola-Malpighi Hospital, University of Bologna, Bologna, Italy; ${ }^{10}$ Unit of Pathology, Luigi Sacco University Hospital, Milan, Italy; ${ }^{11}$ Pathology Section, Spedali Civili Hospital, Brescia, Italy; ${ }^{12}$ Department of Advanced Biomedical Sciences, Federico II University of Naples, Naples, Italy; ${ }^{13}$ Department of Gastroenterology, National Cancer Institute, Aviano, Italy; ${ }^{14}$ Gastroenterology Unit, Department of Life and Environmental Sciences, University of L'Aquila, L'Aquila, Italy; ${ }^{15}$ Department of Medicine and Surgery, University of Salerno, Salerno, Italy and ${ }^{16}$ Department of Systems Medicine, University of Tor Vergata, Rome, Italy

\begin{abstract}
Non-familial small bowel carcinomas are relatively rare and have a poor prognosis. Two small bowel carcinoma subsets may arise in distinct immune-inflammatory diseases (celiac disease and Crohn's disease) and have been recently suggested to differ in prognosis, celiac disease-associated carcinoma cases showing a better outcome, possibly due to their higher DNA microsatellite instability and tumor-infiltrating T lymphocytes. In this study, we investigated the histological structure (glandular vs diffuse/poorly cohesive, mixed or solid), cell phenotype (intestinal vs gastric/pancreatobiliary duct type) and Wnt signaling activation ( $\beta$-catenin and/or SOX-9 nuclear expression) in a series of 26 celiac disease-associated small bowel carcinoma, 25 Crohn's disease-associated small bowel carcinoma and 25 sporadic small bowel carcinoma cases, searching for new prognostic parameters. In addition, non-tumor mucosa of celiac and Crohn's disease patients was investigated for epithelial precursor changes (hyperplastic, metaplastic or dysplastic) to help clarify carcinoma histogenesis. When compared with non-glandular structure and non-intestinal phenotype, both glandular structure and intestinal phenotype were associated with a more favorable outcome at univariable or stage- and microsatellite instability/tumor-infiltrating lymphocyte-inclusive multivariable analysis. The prognostic power of histological structure was independent of the clinical groups while the non-intestinal phenotype, associated with poor outcome, was dominant among Crohn's disease-associated carcinoma. Both nuclear $\beta$-catenin and SOX-9 were preferably expressed among celiac disease-associated carcinomas; however, they were devoid, per se, of prognostic value. We obtained findings supporting an origin of celiac disease-associated carcinoma in SOX-9-positive immature hyperplastic
\end{abstract}

Correspondence: Dr A Vanoli, MD, Department of Molecular Medicine, University of Pavia, Via Forlanini 14, Fondazione IRCCS Policlinico San Matteo, Pavia 27100, Italy.

E-mail: ale.vanol@virgilio.it

${ }^{17}$ These authors are co-contributing first authors.

Data in this study have been partially presented at the 2015 and 2017 annual meetings of the United States and Canadian Academy of Pathology.

Received 6 February 2017; revised 22 March 2017; accepted 23 March 2017; published online 30 June 2017 


\begin{abstract}
crypts, partly through flat $\beta$-catenin-positive dysplasia, and of Crohn's disease-associated carcinoma in a metaplastic (gastric and/or pancreatobiliary-type) mucosa, often through dysplastic polypoid growths of metaplastic phenotype. In conclusion, despite their common origin in a chronically inflamed mucosa, celiac disease-associated and Crohn's disease-associated small bowel carcinomas differ substantially in histological structure, phenotype, microsatellite instability/tumor-infiltrating lymphocyte status, Wnt pathway activation, mucosal precursor lesions and prognosis.

Modern Pathology (2017) 30, 1453-1466; doi:10.1038/modpathol.2017.40; published online 30 June 2017
\end{abstract}

Small bowel carcinomas are relatively rare neoplasms, the majority of which arise sporadically, while few cases are associated with genetic conditions such as familial adenomatous polyposis, Lynch and Peutz-Jeghers syndromes or may develop in immune-mediated inflammatory conditions, such as celiac disease or Crohn's disease. ${ }^{1}$ Several small bowel carcinoma studies are available concerning their histological structure, cell phenotype, nuclear $\beta$-catenin or p53 protein expression and TP53, KRAS, PIK3CA, HER2 or microsatellite instability-related gene mutation/methylation or copy number alteration. ${ }^{2-6}$ These studies, however, deal mostly with sporadic cases or with few cases from other subgroups, with limited information on associated background disease or specific tumor histogenesis, progression and prognosis.

We recently collected a fairly large series of patients with surgically resected celiac diseaseassociated or Crohn's disease-associated small bowel carcinomas and identified a significantly better overall and cancer-specific survival of celiac disease-associated carcinoma patients compared with Crohn's disease-associated carcinoma patients, with no substantial difference between these two carcinoma subsets and sporadic ones, which showed intermediate survival. ${ }^{7}$ Neither stage nor histopathological type and grade according to WHO 2010 classification criteria accounted for such behavior difference, which was, however, attributed to the higher prevalence of microsatellite instability status found in celiac disease-associated carcinoma, in accordance with previous observations. ${ }^{8,9}$ No other molecular change, such as p53 overexpression or TP53 gene mutation, and KRAS or PIK3CA gene mutations, had a significant prognostic impact.

Results of potential clinico-pathological relevance provided by previous studies are those suggesting a prognostic relevance of tumor cell phenotype, ${ }^{3,10,11} \mathrm{a}$ selective tumor association with mucosal metaplastic changes ${ }^{12}$ or dysplastic lesions ${ }^{13-15}$ and a pathogenetic role of molecular changes affecting microsatellite instability status, ${ }^{8,9,16,17}$ Wnt signaling pathway $^{18-20}$ or p53 gene involvement. ${ }^{5}$ In the present study, we reinvestigated our small bowel carcinoma series aiming to (a) look for any prognostic influence of histopathological structure, with special reference to tumor cell cohesion and stromal desmoplasia, and tumor cell phenotype, according to criteria already developed for the evaluation of gastric cancer; ${ }^{21-23}$ (b) expand our analysis to Wnt pathway activation signs such as nuclear $\beta$-catenin and SOX-9 transcription factor expression, including their correlation with microsatellite instability status; and (c) gain new insights into cancer histogenesis, with special reference to the carcinogenetic process at work in the inflamed non-tumor mucosa of celiac disease-associated or Crohn's diseaseassociated small bowel carcinoma patients.

\section{Materials and methods}

\section{Study Population}

This retrospective study included 76 patients with primary non-familial, non-ampullary small bowel carcinoma, including 26 celiac disease-associated small bowel carcinomas, 25 Crohn's diseaseassociated small bowel carcinomas and 25 small bowel carcinomas arising sporadically, ie, without a concomitant intestinal immune-mediated disorder. The patients, enrolled for a former previous clinicopathological study, had surgical resection and complete survival data from 20 tertiary referral Italian Celiac or Inflammatory Bowel Disease Centers participating in the Small Bowel Cancer Italian Consortium. ${ }^{6}$ The main exclusion criteria for all small bowel carcinoma subgroups were Lynch syndrome, Peutz-Jeghers syndrome, familial adenomatous polyposis and juvenile polyposis. Neuroendocrine neoplasms were also excluded. This study was approved by the Ethics Committee of the San Matteo Hospital Foundation of Pavia.

\section{Histology and Immunohistochemistry}

Tissue samples were fixed in $4 \%$ formaldehyde and processed in paraffin wax. Four- $\mu$ m-thick sections were stained with hematoxylin-eosin and all cases were reinvestigated for histological findings. Histologically, small bowel carcinomas were reclassified as (a) glandular type (if $>70 \%$ of the tumor exhibited glandular pattern), (b) diffuse/poorly cohesive cell type (signet ring cell cancers or cancers showing diffusely infiltrating, poorly cohesive cells, dispersed in a frequently desmoplastic stroma as single elements or as small aggregates with little to no gland formation, in $>70 \%$ of the tumor), (c) mixed type (characterized by a combination of both glandular 
and poorly cohesive cell/desmoplastic patterns, with at least $30 \%$ each, within the same tumor) and solid cancers (when they showed almost exclusively a solid or trabecular pattern). ${ }^{21,22,24}$ Among solid cancers, (d) medullary-type cancers (characterized by tumor cells exhibiting prominent infiltration by $\mathrm{T}$ lymphocytes and a pushing margin) were distinguished from the (e) non-medullary solid cases lacking these features. ${ }^{22}$ Four small bowel carcinomas previously classified as 'mucinous carcinomas,7 were included in the glandular (three cases) or mixed (one case) type according to the predominant structure of the neoplasm. All cases were also checked for the presence of adjacent dysplastic lesions and metaplastic features of the uninvolved mucosa.

For immunohistochemistry, four- $\mu$ m-thick sections were incubated at $4{ }^{\circ} \mathrm{C}$ for $18-20 \mathrm{~h}$ with specific antibodies directed against: the gastric foveolar cell marker MUC5AC (monoclonal, clone CLH2, Abcam), the pancreatobiliary duct marker cytokeratin (CK) 7 (monoclonal, clone OV-TL 12/30, Dako), the pyloric and Brunner's gland marker MUC6 (monoclonal, clone CLH5, Novocastra), as well as against intestinal differentiation markers, including the transcription factor CDX2 (monoclonal, clone DAK-CDX2, Dako), the goblet cell marker MUC2 (monoclonal, clone Ccp58, Santa Cruz Biotechnology), CK20 (monoclonal, clone Ks20.8, Dako) and the small bowel brush border marker CD10 (monoclonal, clone 56C6, Dako). In addition, immunoreactions for C-terminal $\beta$-catenin (monoclonal, clone 14/Beta-Catenin, BD); for the transcription factor Sex-determining Region Y-Box 9 (SOX-9, polyclonal, Millipore); for the mismatch repair proteins, including MLH1 (monoclonal, clone ES05, Dako), MSH2 (monoclonal, clone FE11, Dako), MSH6 (monoclonal, clone EP49, Dako) and PMS2 (monoclonal, clone EP51, Dako); for p53 protein (monoclonal, clone DO7, Dako); for the neuroendocrine marker chromogranin-A (monoclonal, clone LK2H10, Ventana) and for CD3 (polyclonal, Dako) and CD8 (polyclonal, Dako) were also performed. Immunoreactions were developed using $0.03 \% 3,3$ ' diaminobenzidine tetrahydrochloride and sections were then counterstained with Harris' hematoxylin.

For the assessment of tumor cell phenotype, only cases with at least $10 \%$ immunoreactive cells were regarded as positive with the exception of CDX-2, for which a cutoff of $20 \%$ was applied. ${ }^{23}$ Cases with nuclear accumulation of C-terminal $\beta$-catenin in at least $10 \%$ of dysplastic/tumor cells was recorded as positive and were also tested for the loss of nuclear expression of $\mathrm{N}$-terminal $\beta$-catenin (monoclonal, clone E247, Abcam). Only relatively strong nuclear SOX-9 immunoreactivity, with the same intensity as the deepest intestinal crypt cells, was regarded as positive. Immunostaining of mismatch repair proteins in neoplastic cells was evaluated as positive or negative. Tumor or dysplastic growths were considered negative when there was complete absence of nuclear staining of neoplastic cells in the presence of an internal positive control. A tumor was classified as having high density of tumor-infiltrating lymphocytes when the mean number of CD3-positive tumorinfiltrating lymphocytes per high-power field was $>15$, as previously reported. ${ }^{7,25}$ Tumor or dysplastic lesions were considered p53 positive when $\geq 50 \%$ of the cells showed strong nuclear p53 immunoreactivity. A central pathology review was performed by two surgical pathologists specialized in gastrointestinal pathology (AV and ES).

\section{Microsatellite Instability Analysis}

Tumor DNA was obtained from formalin-fixed and paraffin-embedded tissues using three representative $8 \mu \mathrm{m}$-thick sections of tumor samples. DNA was extracted after manual microdissection using a QIAamp DNA Formalin-Fixed, Paraffin-Embedded Tissue Kit according to the manufacturer's protocol (Qiagen, Hilden, Germany). Microsatellite instability analysis was performed using a pentaplex panel of monomorphic mononucleotide repeats (BAT25, BAT26, NR-21, NR-22 and NR-24) by the ABI PRISM 310 Genetic Analyzer (Applied Biosystems, Foster City, CA, USA).

\section{MLH1 Methylation Analysis}

MLH1 methylation status was examined by pyrosequencing in small bowel carcinomas exhibiting loss of MLH1 immunohistochemical expression as previously reported. ${ }^{7}$

\section{Statistical Analysis}

The distribution of biomarkers was reported as counts and percentage and Fisher's exact test was used to compare them across clinical groups. Cumulative survival was plotted according to the Kaplan-Meier method. The follow-up extended from the date of surgery to the date of death or last followup. The association candidate prognostic factors and tumor-related death was estimated by means of univariable and multivariable Cox regression. The choice of variables to be included in the multivariable model was decided $a$ priori and was based on the biological knowledge of the tumor. Hazard ratios and their 95\% confidence intervals were computed. The proportional hazard assumption was tested, based on Schoenfeld residuals. Model discrimination was assessed with the Harrell's $C$ statistic (the closer to 1 , the better) and calibration with the shrinkage coefficient (the closer to 1 , the better). A two-sided $P$-value $<0.05$ was considered statistically significant. For post hoc comparisons, Bonferroni's correction applies. Stata 14 (StataCorp, College Station, TX, USA) was used for computation. 


\section{Results}

\section{Histological Classification}

Histological analysis showed predominance of gland-forming tumors over diffuse, mixed glandular/diffuse, medullary or non-medullary solid cases (Table 1 and Figure 1). Survival analysis of these five tumor histotypes gave a trend for worse outcome of diffuse, mixed and solid non-medullary compared with glandular or medullary cases (Figure 2a). When diffuse, mixed and solid histotypes were pooled in a single group of 28 cases (Figure 2b), a significant survival difference emerged for this group in comparison with glandular cases (hazard ratio: 4.93, 2.23-10.89, $P<0.001)$. Multivariable analysis confirmed a significantly improved survival of

Table 1 Histological classification of the 76 small bowel carcinomas investigated

\begin{tabular}{lccc}
\hline Histotype & \multicolumn{3}{c}{ Distribution among clinical groups } \\
\cline { 2 - 4 } & $\begin{array}{c}\text { Celiac disease-associated carcinomas } \\
(\mathrm{n}=26)\end{array}$ & $\begin{array}{c}\text { Crohn's disease-associated carcinomas } \\
(\mathrm{n}=25)\end{array}$ & $\begin{array}{c}\text { Sporadic carcinomas } \\
(\mathrm{n}=25)\end{array}$ \\
\hline Glandular & $14(54 \%)$ & $13(52 \%)$ & $15(60 \%)$ \\
Medullary & $4(15 \%)$ & $1(4 \%)$ & $1(4 \%)$ \\
Solid & $3(11.5 \%)$ & $2(8 \%)$ & $3(12 \%)$ \\
Diffuse & $2(8 \%)$ & $4(16 \%)$ & $1(4 \%)$ \\
Mixed & $3(11.5 \%)$ & $5(20 \%)$ & $5(11 \%)$ \\
\hline
\end{tabular}

No significant histotype distributive difference among the clinical groups was found.
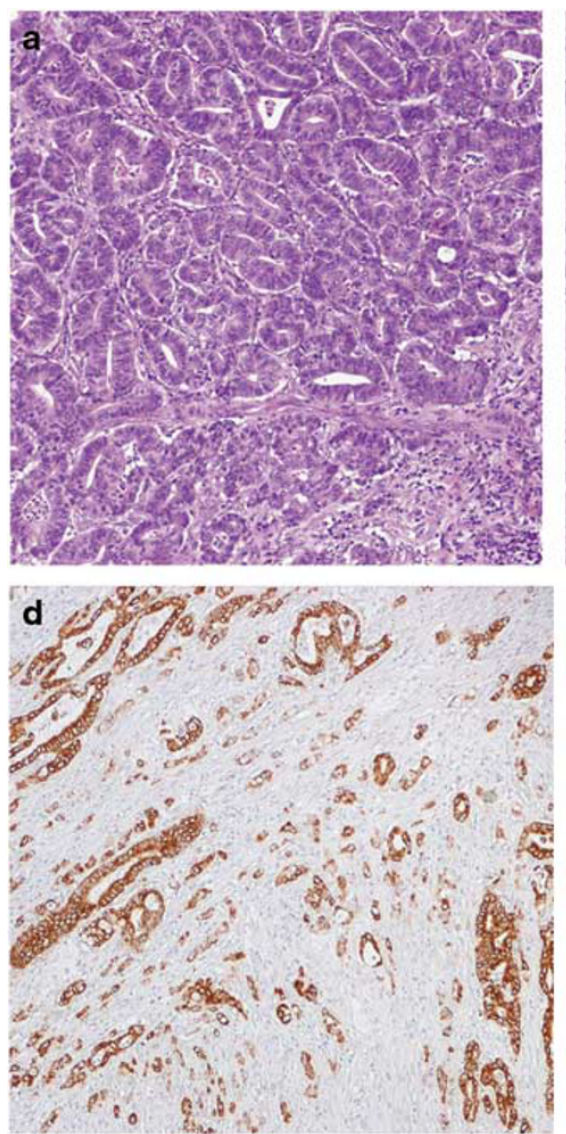
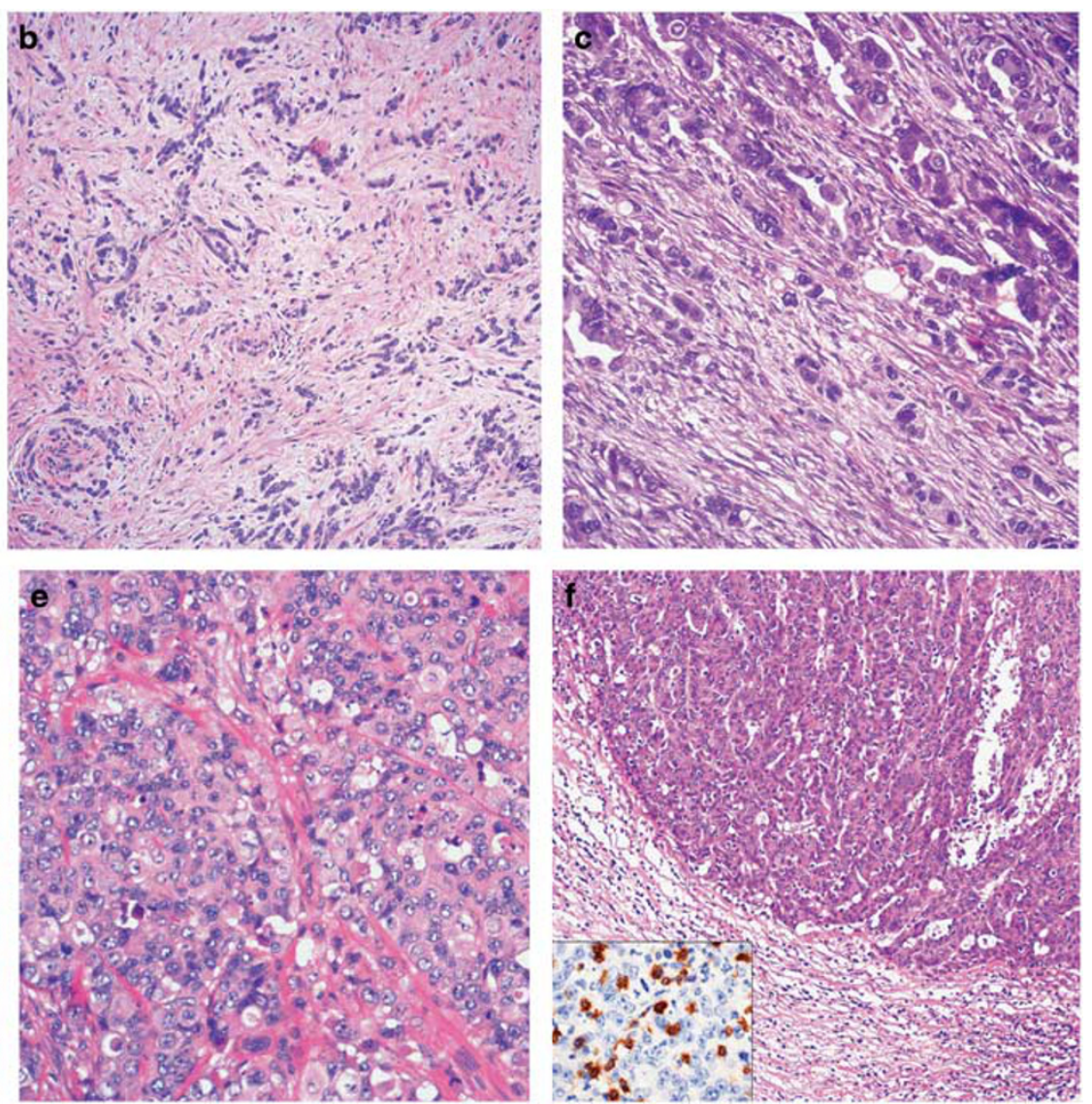

Figure 1 Small bowel carcinoma histotypes. (a) This glandular-type celiac disease-associated carcinoma is predominantly composed of well-formed tubular structures. (b) This diffuse type celiac disease-associated carcinoma is characterized by poorly cohesive cells, dispersed in a desmoplastic stroma as single elements or as small aggregates. (c, d) A mixed type Crohn's disease-associated ileal carcinoma characterized by a combination of both glandular and diffuse patterns within the same tumor. In panel (d), the same Crohn's disease-associated carcinoma expressing the gastric marker MUC5AC in both components. (e) A non-medullary solid-type celiac diseaseassociated carcinoma composed of nests of large, eosinophilic and atypical cells without intratumor T-cell infiltration. (f) This medullarytype celiac disease-associated carcinoma is characterized by solid/trabecular growth of cells, a pushing margin and prominent infiltration by numerous $\mathrm{T}$ lymphocytes (see CD3 immunostaining in the inset). 
a
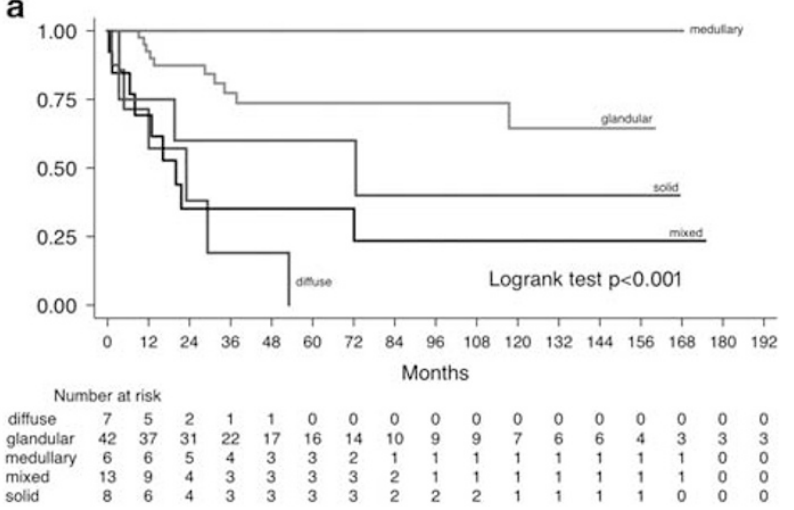

b

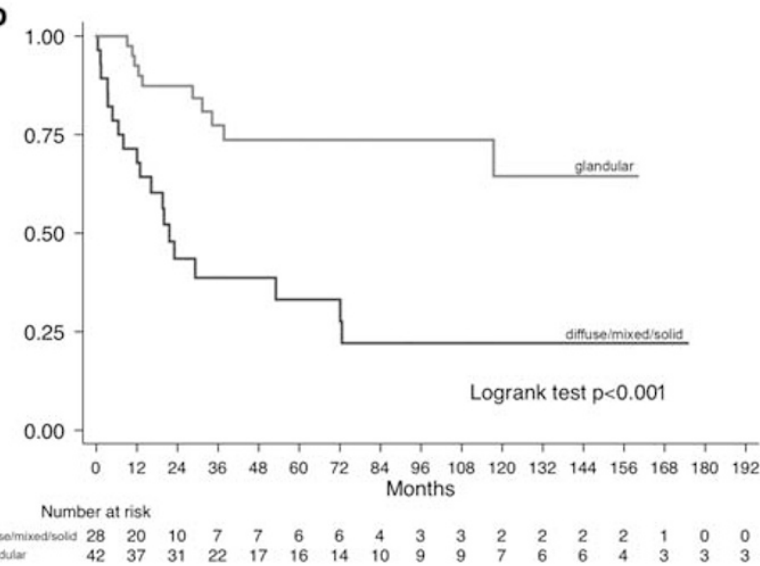

Figure 2 (a) Kaplan-Meier survival estimates for all 76 small bowel carcinoma patients by histotype. Post hoc comparisons (significance after Bonferroni's correction set at $P<0.005$ ): (A) significant differences: diffuse vs glandular: $P<0.001$; diffuse vs medullary: $P=0.003$; glandular vs mixed: $P<0.001$; (B) nonsignificant trends: medullary vs mixed: $P=0.013$; medullary vs solid: $P=0.064$; glandular vs solid: $P=0.088$; (C) non-significant differences: diffuse vs mixed: $P=0.544$; diffuse vs solid: $P=0.170$; mixed vs solid: $P=0.401$; glandular vs medullary: $P=0.207$. Notably, no difference was found between diffuse, mixed and solid histotypes, which were pooled in panel (b). (b) Kaplan-Meier survival estimates for the 70 non-medullary small bowel carcinoma patients by glandular vs diffuse/mixed/solid histotype.

patients with glandular-type small bowel carcinoma, independently of clinical group, tumor-infiltrating lymphocyte density (or microsatellite instability) and stage (Table 2).

\section{Tumor Cell Phenotype}

The distribution of the intestinal phenotypic markers CDX2, MUC2, CK20 or CD10, the gastric marker MUC5AC and the pancreatobiliary duct marker CK7 among small bowel carcinoma subsets is outlined in Table 3, where 39 tumors with essentially intestinal phenotype (ie, positive for CDX2, MUC2, CK20 and/or CD10, whereas negative for both MUC5AC and CK7) were separated from the remaining 37 tumors expressing a metaplastic gastro-pancreatobiliary phenotype, either alone or admixed with the intestinal one (ie, positive for MUC5AC and/or
Table 2 Overall survival by a multivariable Cox model of the 75 small bowel carcinomas patients with complete stage data

\begin{tabular}{lcc}
\hline & Hazard ratio (95\% CI) & P-value \\
\hline $\begin{array}{l}\text { Clinical group } \\
\text { Celiac disease-associated } \\
\text { carcinomas }\end{array}$ & 1.00 (base) & 0.033 \\
$\quad \begin{array}{l}\text { Crohn's disease-associated } \\
\text { carcinomas }\end{array}$ & $4.98(1.35-18.34)$ & $0.016^{\mathrm{a}}$ \\
$\quad$ Sporadic carcinomas & $2.48(0.66-9.29)$ & $0.176^{\mathrm{a}}$, \\
$\begin{array}{l}\text { Stages III-IV vs stages I-II } \\
\text { CD3 }{ }^{+} \text {tumor-infiltrating }\end{array}$ & $9.31(3.30-26.26)$ & $<0.001$ \\
$\begin{array}{l}\text { lymphocytes > 15/high- } \\
\text { power field vs } \leq 15\end{array}$ & $0.15(0.03-0.69)$ & 0.015 \\
$\begin{array}{l}\text { Glandular histotype vs non- } \\
\text { glandular }\end{array}$ & $0.37(0.16-0.84)$ & 0.018 \\
\hline
\end{tabular}

Model: $\quad \operatorname{LR} \quad \operatorname{chi}^{2}(5)=56.72, \quad P$-value $<0.001 ; \quad$ Harrell's $C=0.87$; shrinkage coefficient $=0.91$.

${ }^{\mathrm{a}}$ For post hoc comparisons among the clinical groups, significance after Bonferroni's correction set at 0.017 .

${ }^{b}$ Hazard ratio (95\% CI): $0.50(0.22-1.14), P$-value $=0.098$ vs Crohn's disease-associated small bowel carcinomas.

${ }^{\mathrm{C}}$ Medullary histotype included among non-glandular cases.

CK7 while being positive or negative for CDX2, MUC2, CK20 and CD10). A general predominance of the intestinal phenotype among both celiac diseaseassociated and sporadic cases and of the nonintestinal (gastro-pancreatobiliary or mixed) phenotype among Crohn's disease cases was observed (Figure 3). Intestinal phenotype was more frequently found in glandular $(32 / 42,76 \%)$ compared with diffuse/mixed/solid cases $(6 / 28,21 \%, P<0.001)$. In particular, 11 of the 13 mixed, 4 of the 7 diffuse and 7 of the 8 solid small bowel carcinomas showed gastropancreatobiliary markers expression. Of note, all of the 10 glandular cases with the non-intestinal phenotype occurred in Crohn's disease patients.

Survival analysis showed a favorable influence of individual intestinal markers (especially CDX2 and MUC2) and a worse influence of gastropancreatobiliary markers. The pyloric and Brunner's gland marker MUC6 was found in a small fraction $(16 \%)$ of cases, including 2 celiac disease-associated, 7 Crohn's disease-associated and 3 sporadic small bowel carcinomas, without a significant difference among clinical groups $(P=0.156)$ and without prognostic value (hazard ratio: $0.80,0.30-2.13, P=0.654$ ).

Cumulative markers analysis showed better survival for patients with tumors of essentially intestinal phenotype compared with those expressing nonintestinal markers (in at least $10 \%$ of cells) (Table 3). This finding may contribute to the poor outcome of Crohn's disease-associated small bowel carcinoma patients, most of which $(20 / 25,80 \%)$ showed non-intestinal marker expression. In a stage-, sexand age-inclusive survival analysis of 75 small bowel carcinomas, the favorable influence of intestinal phenotype was retained (hazard ratio: 0.30, 0.14-0.69; $P=0.004$ ), while it lost significance when the clinical group was also added to the model. 
Table 3 Expression of phenotypic markers in 76 small bowel carcinomas: their distribution among clinical groups and prognostic value

\begin{tabular}{|c|c|c|c|c|c|c|}
\hline & \multicolumn{3}{|c|}{ Distribution among the clinical groups } & \multirow{2}{*}{ Total } & \multicolumn{2}{|c|}{ Survival analysis } \\
\hline & $\begin{array}{c}\text { Celiac disease } \\
\text { carcinomas }(\mathrm{n}=26)\end{array}$ & $\begin{array}{c}\text { Crohn's disease } \\
\text { carcinomas }(\mathrm{n}=25)\end{array}$ & $\begin{array}{c}\text { Sporadic } \\
\text { carcinomas }(\mathrm{n}=25)\end{array}$ & & $\begin{array}{l}\text { Hazard ratio ( } 95 \% \\
\text { confidence interval) }\end{array}$ & $\mathrm{P}$-value \\
\hline Cytokeratin 7 & $5(19 \%)^{\mathrm{a}}$ & $15(60 \%)^{b}$ & $4(16 \%)$ & $24(32 \%)$ & $2.72(1.18-6.29)$ & 0.019 \\
\hline MUC5AC & $5(19 \%)$ & $13(52 \%)$ & $5(20 \%)$ & $23(30 \%)$ & $2.54(1.08-5.98)$ & 0.032 \\
\hline $\mathrm{CDX} 2$ & $21(81 \%)^{\mathrm{C}}$ & $9(36 \%)$ & $17(68 \%)$ & $47(62 \%)$ & $0.28(0.13-0.63)$ & 0.002 \\
\hline MUC2 & $18(69 \%)$ & $12(48 \%)$ & $12(48 \%)$ & $42(55 \%)$ & $0.25(0.12-0.53)$ & $<0.001$ \\
\hline Cytokeratin 20 & $13(50 \%)$ & $9(36 \%)^{d}$ & $20(80 \%)$ & $42(55 \%)$ & $1.19(0.57-2.50)$ & 0.635 \\
\hline CD10 & $8(31 \%)$ & $3(12 \%)$ & $11(44 \%)$ & $22(29 \%)$ & $0.66(0.30-1.65)$ & 0.302 \\
\hline $\begin{array}{l}\text { Intestinal } \\
\text { phenotype }\end{array}$ & $17(65 \%)^{\mathrm{e}}$ & $5(20 \%)^{f}$ & $17(68 \%)$ & $39(51 \%)$ & $0.39(0.18-0.81)^{g}$ & 0.012 \\
\hline
\end{tabular}

${ }^{\mathrm{a}} P=0.004$ vs Crohn's disease-associated carcinomas.

$\mathrm{b}_{P}=0.003$ vs sporadic carcinomas.

${ }^{\mathrm{C}} P=0.002$ vs Crohn's disease-associated carcinomas.

$\mathrm{d}_{P}=0.004$ vs sporadic carcinomas.

${ }^{\mathrm{e}} P=0.002$ vs Crohn's disease-associated carcinomas.

$\mathrm{f}_{P}=0.001$ vs sporadic carcinomas.

${ }^{g}{ }_{V s}$ remaining 37 cases expressing gastro-pancreatobiliary markers.

However, in a multivariable analysis, inclusive of stage and microsatellite instability or tumorinfiltrating lymphocytes, the intestinal phenotype remained predictive of improved survival (hazard ratio: $0.35,0.16-0.77, P=0.009)$.

\section{Impact of Microsatellite Instability and Tumor-Infiltrating Lymphocytes on Prognostic Power of Histotype and Phenotype}

In a previous study, ${ }^{7}$ the small bowel carcinoma microsatellite instability status (ascertained by concordant MLH1 nuclear loss, molecular analysis and MLH1 promoter hypermethylation) was found to be associated with improved survival and to correlate with tumor-infiltrating lymphocytes, the likely microsatellite instability antitumor effectors. ${ }^{22,26}$ Thus when we found that both microsatellite instability and high tumor-infiltrating lymphocyte density were segregating among small bowel carcinomas showing glandular or medullary histotype and intestinal phenotype, we investigated for any potential impact of microsatellite instability and/or high tumor-infiltrating lymphocytes on the prognostic power of histotype and phenotype. Separation of 25 cases showing microsatellite instability, 28 cases with high tumor-infiltrating lymphocytes or 19 cases showing both microsatellite instability and high tumor-infiltrating lymphocytes from the remaining small bowel carcinomas gave highly significant survival differences (hazard ratio: 0.22, 0.08-0.64, $P=0.005$; hazard ratio: $0.09,0.02-0.36, P<0.001$ and hazard ratio: $0.26,0.12-0.57 ; \quad P<0.001$, respectively). Survival analysis of the 51 microsatellite stable or the 48 low tumor-infiltrating lymphocyte cases confirmed the importance of histological structure and phenotype with an improved survival of glandular vs diffuse/mixed/ solid cases (hazard ratio: $0.28,0.12-0.63, P=0.002$ among microsatellite stable cases and hazard ratio: $0.40,0.18-0.87, \quad P=0.022$ among low tumorinfiltrating lymphocyte cases, respectively) and of intestinal vs non-intestinal phenotype small bowel carcinoma cases (hazard ratio: 0.50, 0.23-1.08, $P=0.080$ and hazard ratio: $0.41,0.19-0.89$, $P=0.023$, respectively).

\section{Small Bowel Carcinoma Expression of Nuclear $\beta$-Catenin and SOX-9}

As shown in Table 4 and Figures $4 \mathrm{a}$ and b, nuclear $\beta$-catenin expression was significantly concentrated within the celiac disease-associated small bowel carcinoma group, which differed significantly from the remaining small bowel carcinomas. Interestingly, $\mathrm{N}$-terminally directed antibodies failed to recognize nuclear $\beta$-catenin in $17 / 40(42 \%)$ tumors where the protein was easily detected in the nucleus by C-terminal antibodies. Both N- and C-terminal antibodies recognized membranous $\beta$-catenin in normal non-tumor mucosa of the same cases, thus suggesting the possibility of a tumor-selective N-terminal $\beta$-catenin loss. This was found to be unrelated to patient background disease and survival (Table 4). A relationship of our findings with the $\beta$-catenin gene deletion reported by Breuhahn et $a l^{27}$ in some sporadic small bowel carcinomas seems likely. Similar to the nuclear $\beta$-catenin expression, the loss of MLH1 expression (which overlapped perfectly with molecularly assessed microsatellite instability status) was also highly and selectively concentrated among celiac disease-associated small bowel carcinomas ( $P=0.001$ vs remaining small bowel carcinomas). In fact, a correlation was found among the whole small bowel cancer series between the distribution of the two changes $(P<0.001$, Fisher's test).

The nuclear expression of SOX-9, another Wnt-related transcription factor, was significantly 

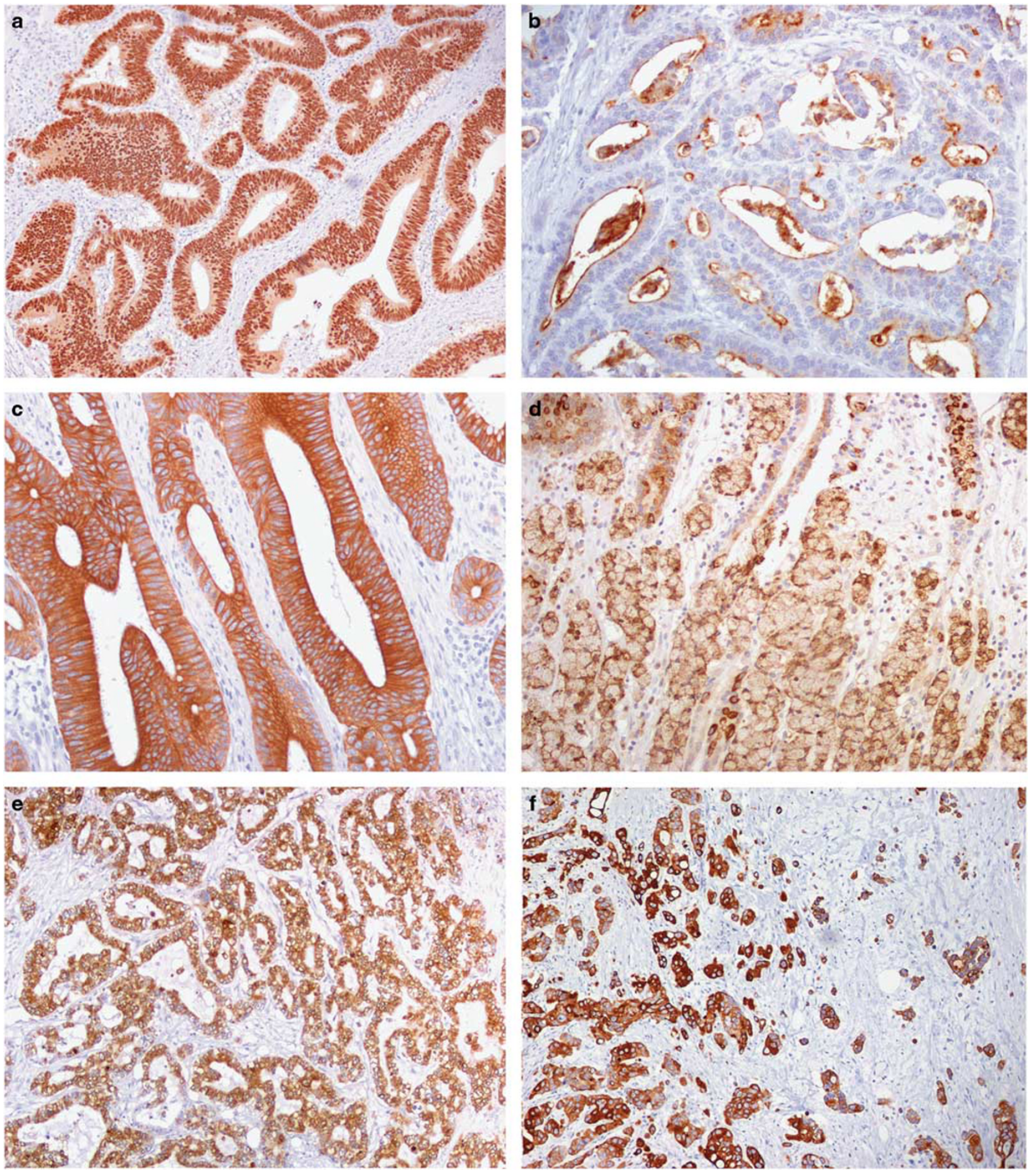

Figure 3 Phenotypic marker expression in small bowel carcinomas. (a) Uniform and intense nuclear positivity for the intestinal transcription factor CDX2 in a glandular-type celiac disease-associated carcinoma. (b) Luminal surface expression of the brush border marker CD10 in a celiac disease-associated carcinoma. (c) A sporadic small bowel carcinoma reactive for the intestinal marker CK20. (d) Signet ring cell Crohn's disease-associated carcinoma reactive for the goblet cell mucin MUC2. (e) A glandular Crohn's disease-associated carcinoma extensively expressing the gastric foveolar marker MUC5AC. (f) A mixed-type Crohn's disease-associated carcinoma reactive for the pancreatobiliary duct marker CK7.

correlated with that of $\beta$-catenin ( $P=0.005$, Fisher's test) and was also highly represented among celiac disease-associated small bowel carcinomas (Figure 4c), although less selectively than $\beta$-catenin. Indeed, no significant difference for SOX-9 expression was found among clinical groups. A trend for SOX-9 to be more extensively expressed in intramucosal or superficial than in deeply invasive tumor growths was noted. 
Univariate survival analysis of the whole series showed a significantly better survival for patients bearing nuclear $\beta$-catenin-positive vs -negative small bowel carcinoma, whereas SOX-9 failed to reveal prognostic relevance. The association of nuclear $\beta$-catenin with more favorable outcome was retained in a stage-, age- and sex-inclusive multivariable model (hazard ratio: $0.32,0.13-0.75 ; P=0.010$ ) or in a stage- and histotype-inclusive model (hazard ratio: $0.29,0.13-0.65 ; P=0.003$ ), while losing significance only when clinical groups were added to the model. However, when survival of 20 small bowel carcinoma patients showing both microsatellite instability and nuclear $\beta$-catenin was compared with that of 20 cases showing nuclear $\beta$-catenin in the absence of microsatellite instability, the latter group had a worse outcome (hazard ratio: 7.14, 2.00-25.00; $P=0.002$ ). In addition, among the 51 microsatellite stable cases, the $20 \beta$-catenin-positive small bowel carcinoma cases lacked any survival difference compared with $31 \beta$-catenin-negative small bowel carcinoma patients (hazard ratio: 0.66, 0.31-1.43; $P=0.296$ ).

\section{Small Bowel Carcinoma-Associated Dysplasia and Other Preneoplastic Changes}

Evidence for residual adenomatous polypoid growth was obtained, within or adjacent to the superficial part of the neoplasm, in 10 of the 25 sporadic small bowel carcinomas, 8 of the 25 Crohn's diseaseassociated carcinomas and only 1 of the 26 celiac disease-associated carcinoma. Focal flat dysplasia, usually adjacent to the invasive cancer focus, was detected in 4 celiac disease-associated, 1 sporadic and 5 Crohn's disease-associated small bowel carcinomas (Figure 4d). Minute dysplastic foci were

Table 4 Small bowel carcinoma molecular alterations: distribution among clinical groups and survival analysis

\begin{tabular}{|c|c|c|c|c|c|c|}
\hline & \multicolumn{3}{|c|}{ Distribution among clinical groups } & \multirow{2}{*}{ Total cases } & \multicolumn{2}{|c|}{ Survival analysis } \\
\hline & $\begin{array}{l}\text { Celiac disease } \\
\text { carcinomas }\end{array}$ & $\begin{array}{l}\text { Crohn's disease } \\
\text { carcinomas }\end{array}$ & $\begin{array}{l}\text { Sporadic } \\
\text { carcinomas }\end{array}$ & & $\begin{array}{l}\text { Hazard ratio (95\% } \\
\text { confidence interval) }\end{array}$ & P-value \\
\hline $\begin{array}{l}\text { Nuclear } \beta \text {-catenin, } \\
\text { C-terminal antibody }\end{array}$ & $24 / 26(92 \%)^{\mathrm{a}}$ & $6 / 24(25 \%)$ & $10 / 25(40 \%)$ & $40 / 75(53 \%)$ & $0.30(0.14-0.63)$ & 0.002 \\
\hline $\begin{array}{l}\text { Nuclear N-terminal } \\
\beta \text {-catenin loss }\end{array}$ & $10 / 26(38 \%)$ & $2 / 24(8 \%)$ & $5 / 25(20 \%)$ & $17 / 75(23 \%)$ & $0.49(0.22-1.12)^{\mathrm{b}}$ & 0.091 \\
\hline $\begin{array}{l}\text { Among C-terminal } \\
\beta \text {-catenin-positive cases }\end{array}$ & $10 / 24(42 \%)$ & $2 / 6(33 \%)$ & $5 / 10(50 \%)$ & $17 / 40(42 \%)$ & $0.85(0.24-2.97)^{\mathrm{C}}$ & 0.796 \\
\hline SOX-9 expression & $20 / 23(87 \%)$ & $11 / 22(50 \%)$ & $14 / 23(51 \%)$ & $45 / 68(66 \%)$ & $0.70(0.31-1.56)$ & 0.380 \\
\hline $\begin{array}{l}\text { Microsatellite instability } \\
\text { Among C-terminal } \\
\beta \text {-catenin-positive cases }\end{array}$ & $\begin{array}{l}17 / 26(65 \%)^{\mathrm{e}} \\
17 / 24(71 \%)^{\mathrm{a}}\end{array}$ & $\begin{array}{r}4 / 25(16 \%) \\
1 / 6(17 \%)\end{array}$ & $\begin{array}{l}4 / 25(16 \%) \\
2 / 10(20 \%)\end{array}$ & $\begin{array}{l}25 / 76(33 \%) \\
20 / 40(50 \%)\end{array}$ & $\begin{array}{l}0.22(0.08-0.64) \\
0.14(0.04-0.50)\end{array}$ & $\begin{array}{l}0.005 \\
0.002\end{array}$ \\
\hline
\end{tabular}

${ }^{a} P<0.001$ vs Crohn's disease-associated carcinomas or sporadic carcinomas.

${ }^{\mathrm{b}}$ Vs remaining small bowel carcinomas.

${ }^{\mathrm{C}} V S$ remaining C-terminal $\beta$-catenin-positive cases.

$\mathrm{d}$ All 17 microsatellite unstable cases also had nuclear $\beta$-catenin among celiac disease-associated carcinomas; as against only one of the 4 among Crohn's disease-associated carcinomas and 2 of the 4 among sporadic carcinomas.

${ }^{\mathrm{e}} P=0.001$ vs Crohn's disease-associated carcinomas or sporadic carcinomas.

Figure 4 Molecular/cellular marker expression and preneoplastic changes. (a, b) Nuclear accumulation of $\beta$-catenin in a celiac diseaseassociated small bowel carcinoma (a), to be compared with a membranous/cytoplasmic reactivity of a Crohn's disease-associated small bowel carcinoma (b). (c) Intense SOX-9 expression in the tumor cell nuclei of a celiac disease-associated carcinoma. (d) Evidence for residual high-grade dysplasia overlying the superficial part of an invasive celiac disease-associated small bowel carcinoma (arrowheads). (e, f) Nuclear SOX-9 (e) and $\beta$-catenin (f) expression in a flat, low-grade, dysplastic lesion of a celiac disease-associated carcinoma case. Note the villous atrophy and, in the inset of panel (e), the extensive nuclear SOX-9 reactivity of hyperplastic crypts adjacent to dysplasia. (g) Same celiac disease-associated small bowel carcinoma case as in panel (d) showing discrepancy in MLH1 expression between the dysplastic (positive) and the underlying, invasive (negative) components. (h) A normal jejunal mucosa adjacent to a sporadic small bowel carcinoma with nuclear SOX-9 expression restricted to the deep-half of the crypts. Note a few enteroendocrine cells with SOX-9-reactive cytoplasm and unreactive nucleus (arrows). (i) SOX-9 nuclear expression in the atrophic mucosa distant from a celiac disease-associated small bowel carcinoma, showing a prominent SOX-9 expansion to involve the upper half of the crypts, the crypt/villous junction and even the surface epithelium. (j) A focus of MUC5AC-positive, high-grade dysplasia of a Crohn's disease-associated small bowel carcinoma case. (k) A dysplastic lesion and an underlying small focus of diffuse type Crohn's disease-associated small bowel carcinoma (arrowheads), both concordantly reactive for CK7. (l) An isolated, 'microscopic' CK7-positive neoplastic lesion with focal stromal invasion in the small bowel mucosa distant from a 'macroscopic' Crohn's disease-associated small bowel carcinoma. (m) Diffuse and intense MUC5AC positivity of a polypoid adenomatous dysplasia adjacent to a Crohn's disease-associated small bowel carcinoma. 
found, in addition, associated with mucosal metaplastic changes in 3 Crohn's disease-associated carcinomas (see below). Total dysplastic lesions are summarized in Table 5, where it appears that nuclear SOX-9 (Figure 4e), found in 23/28 (82\%) of cases investigated, is the molecular marker most widely expressed in dysplasia, irrespective of clinical group, followed by p53 overexpression (15/29, 52\%) and $\beta$-catenin $(10 / 29,34 \%)$ (Figure $4 \mathrm{f})$, with only 2 of the $29(7 \%)$ dysplastic lesions showing MLH1 loss. On the contrary, the latter change occurred in 24/76 $(32 \%)$ carcinomas, including 5 of the cases retaining
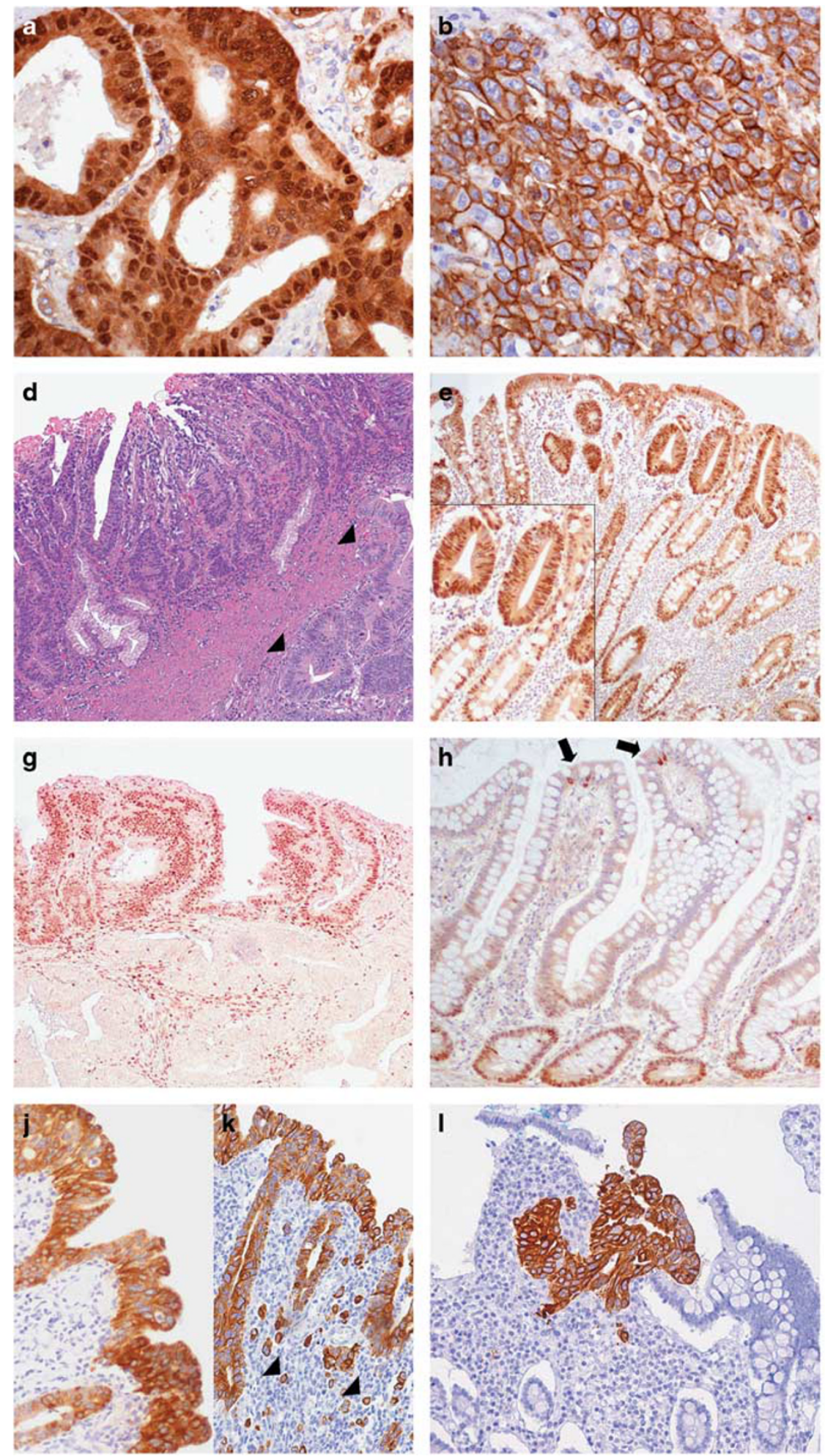
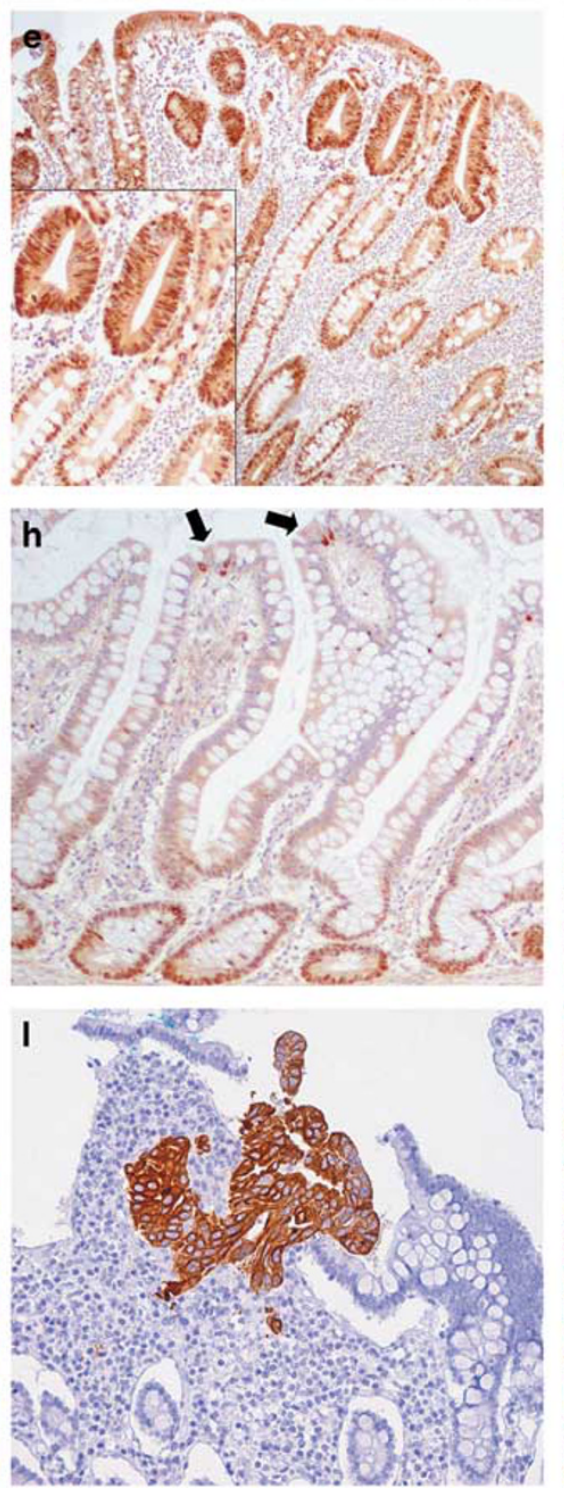
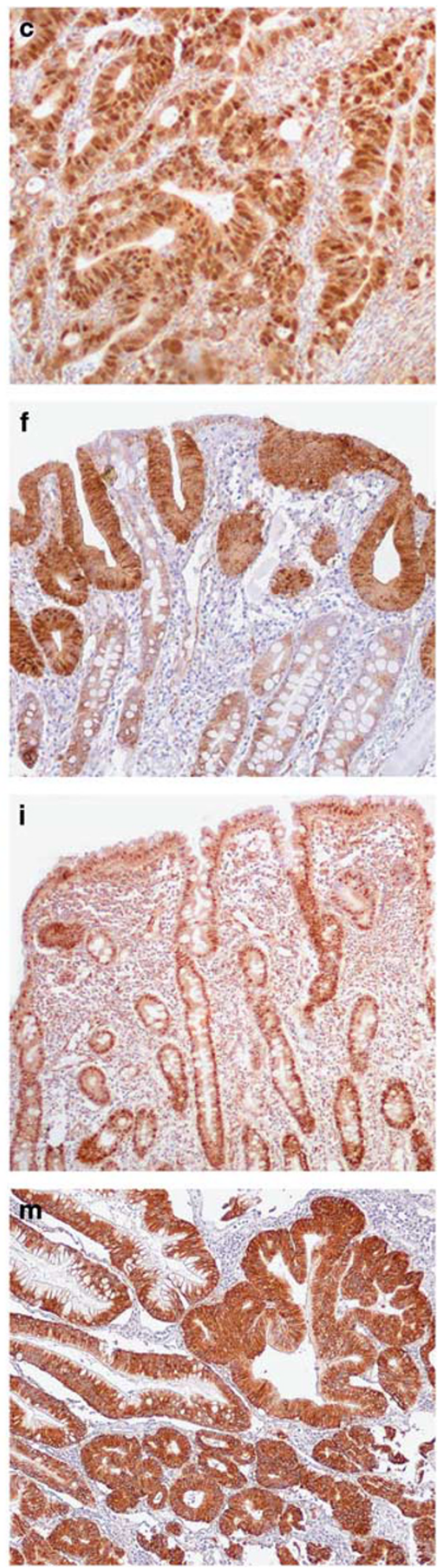
Table 5 Analysis of dysplastic and metaplastic changes associated with small bowel carcinomas

\begin{tabular}{|c|c|c|c|c|}
\hline & Celiac disease carcinomas & Crohn's disease carcinomas & Sporadic carcinomas & Total \\
\hline Dysplasia & $5 / 26(19 \%)^{\mathrm{a}}$ & $16 / 25(64 \%)^{b}$ & $11 / 25(44 \%)^{\mathrm{C}}$ & $32 / 76(42 \%)$ \\
\hline Metaplastic phenotype ${ }^{\mathrm{d}}$ & $0 / 5(0 \%)$ & $14 / 15(93 \%)$ & $2 / 10(20 \%)$ & $16 / 30(53 \%)$ \\
\hline$\beta$-Catenin nuclear expression & $5 / 5(100 \%)$ & $0 / 13(0 \%)$ & $5 / 11(45 \%)$ & $10 / 29(34 \%)$ \\
\hline SOX-9 expression & $5 / 5(100 \%)$ & $10 / 13(77 \%)$ & $8 / 10(80 \%)$ & $23 / 28(82 \%)$ \\
\hline MLH1 loss & $1 / 5(20 \%)$ & 1/14 (7\%) & $0 / 10(0 \%)$ & 2/29 (7\%) \\
\hline p53 overexpression & $4 / 5(80 \%)$ & $7 / 15(47 \%)$ & $4 / 9(44 \%)$ & $15 / 29(52 \%)$ \\
\hline \multicolumn{5}{|l|}{ Non-dysplastic mucosa } \\
\hline Metaplastic phenotype ${ }^{\mathrm{d}}$ & $2 / 25(9 \%)$ & $19 / 22(86 \%)^{\mathrm{e}}$ & $0 / 24(0 \%)$ & $21 / 71(30 \%)$ \\
\hline
\end{tabular}

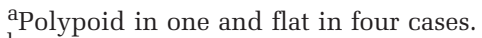

${ }^{b}$ Polypoid in eight, flat in five and minute foci in metaplasia in three cases.

${ }^{\mathrm{C}}$ Polypoid in 10 and flat in 1 case.

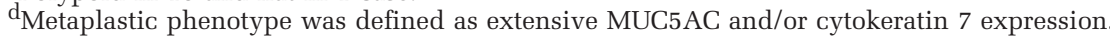

${ }^{\mathrm{e}} P<0.001$ vs either celiac disease-associated carcinomas or sporadic carcinomas.

MLH1 staining in the corresponding dysplasia (Figure $4 \mathrm{~g}$ ). This finding outlines a relatively late appearance of microsatellite instability status during carcinogenesis.

Diffuse or patchy celiac disease lesions, ie, gobletcell poor crypt hyperplasia plus increased intraepithelial lymphocytes, either isolated $(5 / 25)$ or associated with villous atrophy (17/25), were seen in the majority $(22 / 25,88 \%)$ of celiac diseaseassociated small bowel carcinomas, both in canceradjacent and cancer-distant small bowel mucosa. Notably, nuclear SOX-9 expression, normally restricted to the deep lower half of crypts (Figure 4h), showed a prominent expansion to involve the upper half of the crypts in non-tumor mucosa of all 19 celiac disease-associated small bowel carcinomas investigated, sometimes reaching the crypt/villous junction or even to the superficial epithelium covering the flattened mucosa resulting from villous atrophy (Figure 4i). Care was taken to discard chromogranin-A reactive cells, usually showing SOX-9-positive cytoplasm in the absence of nuclear staining and scattered as single elements along the whole crypt-villous unit (Figure 4h). Interestingly, direct continuity of SOX-9-reactive crypt hyperplasia with dysplasia and intramucosal neoplasia was consistently seen (Figure 4e). No relevant change of nuclear $\beta$-catenin expression, which remained restricted to very few cells interposed with Paneth cells within the deepest crypts, was observed in SOX-9-positive crypt hyperplasia. However, intense nuclear $\beta$-catenin appeared in adjacent dysplastic or neoplastic lesions (Figure 4f).

Although no preneoplastic change was identified in non-tumor mucosa of sporadic small bowel carcinomas, metaplastic changes showing the expression of the gastric foveolar marker MUC5AC and/or the ductal pancreatobiliary marker CK7 were extensively represented (86\%) in the chronically inflamed, nonneoplastic mucosa associated with Crohn's diseaseassociated small bowel carcinomas, sometimes coupled with minute dysplastic or neoplastic foci
(Figure 4j). Notably, 93\% of the overt dysplastic lesions associated with Crohn's disease-associated carcinomas showed the expression of CK7 and/or MUC5AC; moreover, the gastro-pancreatobiliary markers were concordantly expressed in metaplastic, dysplastic and invasive components of all but one of the 15 Crohn's disease-associated small bowel carcinomas investigated (Figures $4 \mathrm{k}-\mathrm{m}$ ).

\section{Discussion}

In this study of small bowel carcinoma, retention of intestinal phenotype and gland-forming capacity by tumor cells was associated with better patient survival compared with acquisition of nonintestinal (metaplastic) phenotype or non-glandular histological structure. In addition, a small group of medullary-type microsatellite instability/high tumor-infiltrating lymphocyte cancers, found to have a good prognosis in accordance with previous studies, ${ }^{28-30}$ were distinct from solid non-medullary cancers. As a previous study ${ }^{7}$ showed that both high tumor-infiltrating lymphocyte density and microsatellite instability status were associated with improved survival, the prognostic power of tumor histology and phenotype was confirmed in the microsatellite stable and/or low tumor-infiltrating lymphocyte subpopulations of small bowel carcinoma patients. The prognostic power of glandular histology was also found to retain significance in a multivariable model inclusive of stage, clinical group and tumor-infiltrating lymphocyte density (or microsatellite instability status).

When substituted for glandular histology in the same model, the intestinal phenotype lost prognostic power, which, however, was retained when the clinical groups were eliminated from the model. It should be noted that the group-independent behavior of the glandular histotype fits with its relatively uniform distribution among the groups, while the group-sensitive behavior of the phenotype couples with the high, selective expression of non-intestinal 
markers associated with worse prognosis among Crohn's disease-associated small bowel carcinomas. The latter finding may well contribute to the poor survival shown by Crohn's disease-associated small bowel carcinoma patients. Interestingly, the worse survival of Crohn's disease-associated carcinoma cases reached full statistical significance only with respect to celiac disease-associated carcinoma cases but not to sporadic carcinoma cases. Indeed, the microsatellite instability/high tumor-infiltrating lymphocytes pattern of celiac disease-associated carcinoma is likely to amplify its intestinal phenotype-dependent survival improvement with respect to Crohn's diseaseassociated small bowel carcinoma cases.

The histotype and phenotype approaches proposed here offer new tools for small bowel carcinoma prognostic evaluation, and these should be added to the clinical group characterization and microsatellite instability status suggested by our previous investigations. ${ }^{7}$ Notably, while both histotype and phenotype proved to be prognostic factors independent of microsatellite instability and tumorinfiltrating lymphocyte density, only histotype was fully independent of clinical groups.

As expected, all small bowel carcinomas with medullary-type histology, ie, a solid structure with well-demarcated 'pushing' borders, had high tumorinfiltrating lymphocytes. Five of the six cases also had microsatellite instability status as a likely cause of their increased tumor-infiltrating lymphocyte density. Given the difficulty in separating, on purely histological grounds, gut medullary from lymphoepithelioma-like cancer, ${ }^{31}$ often associated with Epstein-Barr virus infection, a separate investigation tested the single microsatellite stable case with Epstein-Barr virus-encoded small RNAs in situ hybridization, finding it to be diffusely positive. $^{32}$

Similar to microsatellite instability, the nuclear accumulation of $\beta$-catenin was also found to be highly prevalent among celiac disease-associated small bowel carcinomas and to be apparently associated with improved survival. This seemed surprising as, unlike microsatellite instability, nuclear $\beta$-catenin expression has been reported by studies of other neoplasms to imply a less than favorable prognostic influence. ${ }^{33-35}$ However, when among $\beta$-catenin-positive small bowel carcinomas, microsatellite unstable and microsatellite stable cases were compared or among microsatellite stable carcinomas, $\beta$-catenin-positive and -negative cases were compared, the lack of favorable prognostic influence of $\beta$-catenin itself, in the absence of microsatellite instability, became evident. The distributive association we found, especially among celiac disease-associated carcinomas, between nuclear $\beta$-catenin expression and the prognostic favorable microsatellite instability status, might account for this misleading prognostic influence of $\beta$-catenin. Notably, such an association is at variance with colorectal cancer findings, where nuclear $\beta$-catenin expression, highly prevalent among microsatellite stable cases, due to CTNNB1 or APC point mutations, has been reported to be rare in microsatellite unstable cases. ${ }^{36}$ Instead of point mutations, large CTNNB1 N-terminal deletions have been reported to stabilize $\beta$-catenin in $20 \%$ of sporadic small bowel carcinomas, ${ }^{27}$ a finding we confirmed by selective $\mathrm{N}$ - vs C-terminal $\beta$-catenin immunohistochemistry in our sporadic small bowel carcinomas and extended to about $40 \%$ of celiac diseaseassociated small bowel carcinomas. Whether additional stabilization mechanisms may account for the very high celiac disease-associated small bowel carcinoma rate of nuclear $\beta$-catenin (around 90\%) and for its association with microsatellite instability status, it remains to be further investigated. On the other hand, the lack of $B R A F$ mutations and the lack of correlation between KRAS mutations and microsatellite instability found in previous small bowel carcinoma studies $^{5,7}$ rule out a role of these oncogenes in inducing MLH1 gene methylation and, consequently, microsatellite instability status, as has been shown to occur in colorectal cancers. ${ }^{37,38}$ Thus more work is needed to explain the unusual association between microsatellite instability status and Wnt activation among celiac disease-associated small bowel carcinomas.

We found that another Wnt-related protein, the SOX-9 transcription factor, was also highly expressed in small bowel carcinomas, with a distribution comparable to that of nuclear $\beta$-catenin, at least among celiac disease-associated small bowel carcinomas. This finding seems relevant, as SOX-9 high expression has been reported in a number of cancers, including colorectal, gastric, pancreatic, hepatocellular, brain, lung and prostate cancers. ${ }^{39}$ In some of these cancers, SOX-9 expression was found to be associated with tumor progression, invasion and metastasis, a pattern not seen in our small bowel carcinomas. However, an important SOX-9 role in carcinogenesis has also been proposed, ${ }^{39}$ at least in part through Wnt activation via frizzled and LRP receptor overexpression. ${ }^{40,41}$ In keeping with this hypothesis, SOX-9 overexpression has also been reported in some precancerous conditions, including metaplastic and dysplastic lesions of chronic Helicobacter pylori gastritis, ${ }^{42,43}$ chronic bladder injury ${ }^{44}$ and early stages of colorectal tumorigenesis, ${ }^{39}$ as well as in 'acute' untreated adult celiac disease. ${ }^{45}$

This led us to investigate non-tumor mucosa of small bowel carcinoma-bearing patients for SOX-9 expression. In most celiac disease-associated small bowel carcinoma cases, we frequently observed signs of persistent mucosal damage, including atrophy of the villi, excessive intraepithelial $\mathrm{T}$ lymphocyte infiltration and goblet cell-poor and relatively immature crypt hyperplasia, not unlike those reported in biopsies of some non-neoplastic adult celiac disease patients, even when under gluten-free diet. $^{46}$ Notably, multifocal extension of nuclear SOX-9 expression, usually involving the upper half 
of the hyperplastic crypts was found in most celiac disease cases investigated, even in the mucosa at a distance from the neoplasm. By itself, the topographic continuity we observed at some foci between SOX-9-positive crypt hyperplasia and intramucosal dysplastic or cancerous growths may be suggestive for a histogenetic link between such lesions.

A role for SOX-9 in the mucosa repair process of persistent celiac disease lesions seems conceivable considering its known role in intestinal mucosal repair from other types of damage. ${ }^{47}$ The SOX-9activating role of $\mathrm{NF}-\mathrm{kB}$ transcription factor, as ascertained, for instance, in experimental $H$. pylori gastritis, ${ }^{43}$ seems especially important. Indeed, NF- $\mathrm{KB}$ activity, in turn activated by pro-inflammatory cytokines, has been shown to be enhanced in many immune-inflammatory processes, including inflammatory bowel diseases and related carcinogenesis, ${ }^{48}$ as well as celiac disease. ${ }^{49}$ In fact, a switch from the known SOX-9 role in adult stem cell modulation to cancer stem cell activation, often chronic injury promoted and involving constitutive oncogene activation or oncosuppressor gene silencing, has been suggested by several studies. ${ }^{39,44,50-52}$

Our evidence of polypoid adenomatous remnants in the superficial part of Crohn's disease-associated and sporadic small bowel carcinomas supports the hypothesis that the adenoma-carcinoma sequence, well known from colorectal neoplasms, is also operative for these two types of small bowel carcinoma. $^{14,15,53}$ In celiac disease-associated carcinomas, we obtained evidence for a possible adenomatous polyp origin in only a single case, thus confirming the previous finding ${ }^{54}$ of a substantial lack of such lesions in celiac disease cases, while we found four celiac disease-associated carcinomas with flat dysplasia reactive for both nuclear $\beta$-catenin and SOX-9.

Metaplastic changes showing the same gastric and/or pancreatobiliary phenotype as found in the associated cancer were frequent in dysplastic or nondysplastic mucosa adjacent to Crohn's diseaseassociated carcinomas, though not to celiac diseaseassociated or sporadic carcinomas. This finding may suggest a precancerous role of such lesions in Crohn's disease. Thus two distinct histogenetic processes seem at work for celiac diseaseassociated and Crohn's disease-associated small bowel carcinomas, starting from immature crypt hyperplasia or epithelial metaplasia, respectively.

Although a more extensive, prospective and systematic search for hyperplastic, metaplastic and dysplastic lesions is warranted for a better understanding of small bowel carcinoma histogenesis in the two types of immune-inflammatory conditions we studied, the present findings may indicate promising lines of investigation to identify preneoplastic lesions of potential help in early cancer diagnosis.

\section{Acknowledgments}

Small Bowel Cancer Italian Consortium Investigators: Sandro Ardizzone (Gastroenterology, Luigi Sacco University Hospital, Milan, Italy); Marco Astegiano (General and Specialistic Surgery, Città della Salute e della Scienza-Molinette Hospital, Turin, Italy), Giacomo Caio (Division of Gastroenterology, Sant'Orsola-Malpighi Hospital, University of Bologna, Bologna, Italy), Antonio Calabrò (Department of Experimental and Clinical Biomedical Sciences, University of Florence, Florence, Italy), Vincenzo Canzonieri (Departments of Pathology, National Cancer Institute, Aviano, Italy), Roberta Cerutti (Department of Surgical and Morphological Sciences, University of Insubria, Varese, Italy), Antonio Ciardi (Department of Radiological, Oncological, Pathological Sciences, Umberto I Hospital, La Sapienza University, Rome, Italy), Luigi Coppola (Unit of Pathologic Anatomy, San Filippo Neri Hospital, Rome, Italy), Renata D'Incà (Gastroenterology Section, Department of Surgery, Oncology and Gastroenterology, University of Padua, Padua, Italy), Luca Elli (Center for Prevention and Diagnosis of Celiac Disease, Ca' Granda-Ospedale Maggiore Policlinico, Milan, Italy), Stefano Ferrero (Department of Biomedical, Surgical and Dental Sciences, University of Milan, Milan, Italy), Ada M. Florena (Institute of Pathologic Anatomy, Giaccone University Hospital, University of Palermo, Palermo, Italy), Daniela Furlan (Department of Surgical and Morphological Sciences, University of Insubria, Varese, Italy), Antonino G. Giannone (Institute of Pathologic Anatomy, Giaccone University Hospital, University of Palermo, Palermo, Italy), Paolo Giuffrida (Department of Internal Medicine, IRCCS San Matteo Hospital, University of Pavia, Pavia, Italy), Maria C. Macciomei (Pathology Unit, San Camillo-Forlanini Hospital, Rome, Italy), Antonio Maccioni (Pathology Unit, SS. Trinità Hospital, Cagliari, Italy), Giovanni Monteleone (Department of Systems Medicine, University of Tor Vergata, Rome, Italy), Paola Migliora (Unit of Pathological Anatomy, Sant'Andrea Hospital, Vercelli, Italy), Augusto Orlandi (Department of Biopathology and Image Diagnostics, University of Tor Vergata, Rome, Italy), Claudio Papi (Unit of Inflammatory Bowel Diseases, San Filippo Neri Hospital, Rome, Italy), Vittorio Perfetti (Unit of Internal Medicine, S.S. Annuziata Hospital of Varzi, Pavia, Italy), Luca Reggiani Bonetti (Section of Pathology, Department of Diagnostic Medicine and Public Health, University of Modena and Reggio Emilia, Modena, Italy), Aroldo Rizzo (Unit of Pathology, Cervello Hospital, Palermo, Italy), Marianna Salemme (Pathology Section, Spedali Civili Hospital, Brescia, Italy), Giancarlo Sandri (Clinical Nutrition Unit, Sant'Eugenio Hospital, Rome, Italy), Gianluca Sampietro (Inflammatory Bowel Disease Surgery, Luigi Sacco University Hospital, Milan, Italy), Giuseppe Santeusanio (Department of Biopathology and Image Diagnostics, University of Tor 
Vergata, Rome, Italy), Donatella Santini (Division of Pathology, Sant'Orsola-Malpighi Hospital, Bologna, Italy), Marco Silano (Unit of Human Nutrition and Health, Istituto Superiore di Sanità, Rome, Italy), Gaspare Solina (Units of General Surgery, Cervello Hospital, Palermo, Italy), Francesco Tonelli (Department of Surgery and Translational Medicine, University of Florence, Florence, Italy), Davide Trapani (Department of Surgical and Morphological Sciences, University of Insubria, Varese, Italy), Paolo Usai (Department of Internal Medicine, University of Cagliari, Cagliari, Italy).

This work was supported by grants from the San Matteo Hospital Foundation, Pavia, Italy. MM is supported by a fellowship from the San Matteo Hospital Foundation.

\section{Disclosure/conflict of interest}

The authors declare no conflict of interest.

\section{References}

1 Aparicio T, Svrcek M, Zaanan A, et al. Small bowel adenocarcinoma phenotyping, a clinicobiological prognostic study. Br J Cancer 2013;109:3057-3066.

2 Chen ZM, Wang HL. Alteration of cytokeratin 7 and cytokeratin 20 expression profile is uniquely associated with tumorigenesis of primary adenocarcinoma of the small intestine. Am J Surg Pathol 2004;28:1352-1359.

3 Jun SY, Eom DW, Park H, et al. Prognostic significance of CDX2 and mucin expression in small intestinal adenocarcinoma. Mod Pathol 2014;27:1364-1374.

4 Laforest A, Aparicio T, Zaanan A, et al. ERBB2 gene as a potential therapeutic target in small bowel adenocarcinoma. Eur J Cancer 2014;50:1740-1746.

5 Alvi MA, McArt DG, Kelly P, et al. Comprehensive molecular pathology analysis of small bowel adenocarcinoma reveals novel targets with potential for clinical utility. Oncotarget 2015;6:20863-20874.

6 Warth A, Kloor M, Schirmacher P, et al. Genetics and epigenetics of small bowel adenocarcinoma: the interactions of CIN, MSI, and CIMP. Mod Pathol 2011;24:564-570.

7 Vanoli A, Di Sabatino A, Furlan D, et al. Small Bowel Carcinomas in Coeliac or Crohn's Disease: Clinicopathological, Molecular and Prognostic Features. A Study from the Small Bowel Cancer Italian Consortium. J Crohns Colitis 2017 Feb 24. doi: 10.1093/ecco-jcc/ jjx031. [e-pub ahead of print].

8 Potter DD, Murray JA, Donohue JH, et al. The role of defective mismatch repair in small bowel adenocarcinoma in celiac disease. Cancer Res 2004;64:7073-7077.

9 Diosdado B, Buffart TE, Watkins R, et al. Highresolution array comparative genomic hybridization in sporadic and celiac disease-related small bowel adenocarcinomas. Clin Cancer Res 2010;16:1391-1401.

10 Ushiku T, Arnason T, Fukayama M, et al. Extraampullary duodenal adenocarcinoma. Am J Surg Pathol 2014;38:1484-1493.

11 Xue Y, Vanoli A, Balci S, et al. Non-ampullaryduodenal carcinomas: clinicopathologic analysis of 47 cases and comparison with ampullary and pancreatic adenocarcinomas. Mod Pathol 2017;30:255-266.
12 Whitcomb E, Liu X, Xiao SY. Crohn enteritis-associated small bowel adenocarcinomas exhibit gastric differentiation. Hum Pathol 2014;45:359-367.

13 Bruno CJ, Batts KP, Ahlquist DA. Evidence against flat dysplasia as a regional field defect in small bowel adenocarcinoma associated with celiac sprue. Mayo Clin Proc 1997;72:320-322.

14 Genta RM, Feagins LA. Advanced precancerous lesions in the small bowel mucosa. Best Pract Res Clin Gastroenterol 2013;27:225-233.

15 Svrcek M, Piton G, Cosnes J, et al. Small bowel adenocarcinomas complicating Crohn's disease are associated with dysplasia: a pathological and molecular study. Inflamm Bowel Dis 2014;20:1584-1592.

16 Brücher BL, Geddert H, Langner C, et al. Hypermethylation of hMLH1, HPP1, p14(ARF), p16(INK4A) and APC in primary adenocarcinomas of the small bowel. Int J Cancer 2006;119:1298-1302.

17 Bergmann F, Singh S, Michel S, et al. Small bowel adenocarcinomas in celiac disease follow the CIM-MSI pathway. Oncol Rep 2010;24:1535-1539.

18 Sakamoto H, Mutoh H, Miura Y, et al. SOX9 is highly expressed in nonampullary duodenal adenoma and adenocarcinoma in humans. Gut Liver 2013;7:513-518.

19 Suzuki H, Hirata Y, Suzuki N, et al. Characterization of a new small bowel adenocarcinoma cell line and screening of anti-cancer drug against small bowel adenocarcinoma. Am J Pathol 2015;185:550-562.

20 Yuan W, Zhang Z, Dai B, et al. Whole-exome sequencing of duodenal adenocarcinoma identifies recurrent Wnt/ $\beta$-catenin signaling pathway mutations. Cancer 2016;122:1689-1696.

21 Carneiro F, Seixas M, Sobrinho-Simões M. New elements for an updated classification of the carcinomas of the stomach. Pathol Res Pract 1995;191:571-584.

22 Chiaravalli AM, Klersy C, Vanoli A, et al. Histotypebased prognostic classification of gastric cancer. World J Gastroenterol 2012;18:896-904.

23 Solcia E, Klersy C, Vanoli A, et al. The contribution of cell phenotype to the behavior of gastric cancer. Gastric Cancer 2013;16:462-471.

24 Chiaravalli AM, Cornaggia M, Furlan D, et al. The role of histological investigation in prognostic evaluation of advanced gastric cancer. Analysis of histological structure and molecular changes compared with invasive pattern and stage. Virchows Arch 2001;439: 158-169.

25 Chiaravalli AM, Feltri M, Bertolini V, et al. Intratumour $\mathrm{T}$ cells, their activation status and survival in gastric carcinomas characterised for microsatellite instability and Epstein-Barr virus infection. Virchows Arch 2006;448:344-353.

26 Guidoboni M, Gafà R, Viel A, et al. Microsatellite instability and high content of activated cytotoxic lymphocytes identify colon cancer patients with a favorable prognosis. Am J Pathol 2001;159:297-304.

27 Breuhahn K, Singh S, Schirmacher P, et al. Large-scale $\mathrm{N}$-terminal deletions but not point mutations stabilize beta-catenin in small bowel carcinomas, suggesting divergent molecular pathways of small and large intestinal carcinogenesis. J Pathol 2008;215:300-307.

28 Minamoto $\mathrm{T}$, Mai $\mathrm{M}$, Watanabe $\mathrm{K}$, et al. Medullary carcinoma with lymphocytic infiltration of the stomach. Clinicopathologic study of 27 cases and immunohistochemical analysis of the subpopulations of infiltrating lymphocytes in the tumor. Cancer 1990;66:945-952. 
29 Friedman K, Brodsky AS, Lu S, et al. Medullary carcinoma of the colon: a distinct morphology reveals a distinctive immunoregulatory microenvironment. Mod Pathol 2016;29:528-541.

30 Brcic I, Cathomas G, Vanoli A, et al. Medullary carcinoma of the small bowel. Histopathology 2016;69: 136-140.

31 Chetty R. Gastrointestinal cancers accompanied by a dense lymphoid component: an overview with special reference to gastric and colonic medullary and lymphoepithelioma like carcinomas. J Clin Pathol 2012;65: 1062-1065.

32 Vanoli A, Di Sabatino A, Biancone L, et al. Small bowel Epstein-Barr virus-positive lympho-epithelioma-like carcinoma in Crohn's disease. Histopathology 2017;70: 837-839.

33 Srivastava S, Thakkar B, Yeoh KG, et al. Expression of proteins associated with hypoxia and Wnt pathway activation is of prognostic significance in hepatocellular carcinoma. Virchows Arch 2015;466:541-548.

34 Chen Z, He X, Jia M, et al. $\beta$-catenin overexpression in the nucleus predicts progress disease and unfavourable survival in colorectal cancer: a meta-analysis. PLoS ONE 2013;8:e63854.

$35 \mathrm{Li}$ LF, Wei ZJ, Sun $\mathrm{H}$, et al. Abnormal $\beta$-catenin immunohistochemical expression as a prognostic factor in gastric cancer: a meta-analysis. World J Gastroenterol 2014;20:12313-12321.

36 Panarelli NC, Vaughn CP, Samowitz WS, et al. Sporadic microsatellite instability-high colon cancers rarely display immunohistochemical evidence of Wnt signaling activation. Am J Surg Pathol 2015;39:313-317.

37 Fang M, Ou J, Hutchinson L, et al. BRAF oncoprotein functions through the transcriptional repressor MAFG to mediate the $\mathrm{CpG}$ island methylator phenotype. Mol Cell 2014;55:904-915.

38 Serra RW, Fang M, Park SM, et al. A KRAS-directed transcriptional silencing pathway that mediates the CpG island methylator phenotype. eLife 2014;3:e02313.

39 Matheu A, Collado M, Wise C, et al. Oncogenicity of the developmental transcription factor SOX9. Cancer Res 2012;72:1301-1315.

40 Leung CO, Mak WN, Kai AK, et al. SOX9 confers stemness properties in hepatocellular carcinoma through Frizzled-7 mediated Wnt/ $\beta$-catenin signaling. Oncotarget 2016;7:29371-29386.

41 Ma F, Ye H, He HH, et al. SOX9 drives WNT pathway activation in prostate cancer. J Clin Invest 2016;126: 1745-1758.
42 Sashikawa Kimura M, Mutoh H, Sugano K. SOX9 is expressed in normal stomach, intestinal metaplasia, and gastric carcinoma in humans. J Gastroenterol 2011;46:1292-1299.

43 Serizawa T, Hirata Y, Hayakawa Y, et al. Gastric metaplasia induced by Helicobacter pylori is associated with enhanced SOX9 expression via interleukin-1 signaling. Infect Immun 2015;84:562-572.

44 Ling S, Chang X, Schultz L, et al. An EGFR-ERK-SOX9 signaling cascade links urothelial development and regeneration to cancer. Cancer Res 2011;71:3812-3821.

45 Senger S, Sapone A, Fiorentino MR, et al. Celiac disease histopathology recapitulates hedgehog downregulation, consistent with wound healing processes activation. PLoS ONE 2015;10:e0144634.

46 Lanzini A, Lanzarotto F, Villanacci V, et al. Complete recovery of intestinal mucosa occurs very rarely in adult coeliac patients despite adherence to glutenfree diet. Aliment Pharmacol Ther 2009;29:1299-1308.

47 Roche KC, Gracz AD, Liu XF, et al. SOX9 maintains reserve stem cells and preserves radioresistance in mouse small intestine. Gastroenterology 2015;149: 1553-1563.

48 Hartnett L, Egan LJ. Inflammation, DNA methylation and colitis-associated cancer. Carcinogenesis 2012;33: 723-731.

49 Fernandez-Jimenez N, Castellanos-Rubio A, PlazaIzurieta L, et al. Coregulation and modulation of NFкB-related genes in celiac disease: uncovered aspects of gut mucosal inflammation. Hum Mol Genet 2014;23:1298-1310.

50 Aleman A, Adrien L, Lopez-Serra L, et al. Identification of DNA hypermethylation of SOX9 in association with bladder cancer progression using $\mathrm{CpG}$ microarrays. Br J Cancer 2008;98:466-473.

51 Sun L, Mathews LA, Cabarcas SM, et al. Epigenetic regulation of SOX9 by the NF- $\mathrm{NB}$ signaling pathway in pancreatic cancer stem cells. Stem Cells 2013;31: 1454-1466.

52 Liu C, Liu L, Chen X, et al. SOX9 regulates self-renewal and tumorigenicity by promoting symmetrical cell division of cancer stem cells in hepatocellular carcinoma. Hepatology 2016;64:117-129.

53 Perzin KH, Bridge MF. Adenomas of the small intestine: a clinicopathologic review of 51 cases and a study of their relationship to carcinoma. Cancer 1981;48: 799-819.

54 Rampertab SD, Forde KA, Green PH. Small bowel neoplasia in coeliac disease. Gut 2003;52:1211-1214. 